\title{
Measurement of aorta sacral promontory distance in females undergoing computed tomography
}

\begin{abstract}
Introduction: In context of Nepal, pelvic organ prolapse prevalence is estimated at $10 \%$ among women of reproductive age and $24 \%$ among post menopausal women. This study was carried out to measure the aorta sacral promontory distance among the females referred for contrast enhanced CT abdomen in a tertiary hospital.

Methodology: This prospective study was performed in a tertiary hospital in Nepal. Data were collected over the period of 4 months from July to October 2019 with total of 140 patients who underwent Contrast Enhanced CT abdomen. The age, height and weight of the patients were noted. In this study, aorta sacral promontory distance was determined.

Results: The mean age was 48.20 years, mean BMI was 23.91 and mean aorta sacral promontory (APT) distance was $4.57 \pm 0.90 \mathrm{~cm}$. Increased in age was correlated with decrease in APT distance but there was no correlation between BMI and APT distance. According to this study, APT distance was decreased in elderly female patients but no significant change in BMI and APT.

Conclusion: In this study using CT, we found that as the age increased, APT distance decreased and there was no significant change in relation to BMI. Therefore, during sacral colpopexy, the surgeon should be careful and consider the aorta sacral promontory distance in the elder female patients while performing dissection.
\end{abstract}

Keywords: aorta sacral promontory distance, pelvic organ prolapse, sacral colpopexy
Volume 7 Issue I - 2020

\section{Pandit R, Joshi BR, Chand RB}

Department of Radiology and Imaging, Tribhuwan University, Nepal

Correspondence: Birendra Raj Joshi, Department of Radiology and Imaging, Tribhuwan University, Maharajgunj, Kathmandu, Nepal,Tel 977984I70I333.

Email swaslav@gmail.com

Received: December 18, 2019 | Published: January 22, 2020

\section{Introduction}

All the pelvic organs are supported by complex "hammock" muscles, ligaments and fibers that attach to the bony anatomy of the pelvis. When these are weakened those organ can drop. A prolapse happens when the pelvis muscles and tissues can no longer support these organs because the muscles and tissues are weak or damaged. This causes one or more pelvic organs to drop or press into or out of the vagina. Pelvic organ prolapse is a downward descent of female pelvic organs, including the bladder, uterus and the small or large bowel, resulting in protrusion of the vagina, uterus or both. Prolapse development can be attributed to several factors, including vaginal child birth, advancing age and obesity. Pelvic organ prolapse disorder are more common in elderly female population. Women who had vaginal delivery have a higher incidence of pelvic organ prolapse than women who have undergone caesarean delivery. ${ }^{1}$ Sacral colpopexy, a surgical technique for repairing pelvic organ prolapse in women is consider as the gold standard procedure for pelvic organ prolapse. ${ }^{2} \mathrm{~A}$ higher BMI have been associated with increased prevalence of pelvic floor disorder. ${ }^{3}$ Some studies have demonstrated that with increase in age, aorta enlarges and elongates. ${ }^{4}$ During sacral colpopexy, likelihood of a decreased aorta sacral promontory (APT) distance in elderly and hypertensive patients require careful identification of aorta bifurcation and sacral promontory before dissection. ${ }^{4}$ In context of Nepal, pelvic organ prolapse prevalence is estimated at $10 \%$ among women of reproductive age and $24 \%$ among post menopausal women. ${ }^{5}$

During sacral colpopexy, surgeon should be familiar with the proximity of nearby vessels to avoid the complication of iliac artery, veins and aorta bifurcation. ${ }^{6-8}$ Moreover, presacral haemorrhage incurred during dissection of sacral promontory is one of the most feared complication of sacral colpopexy. Vascular variation in location of the level of the bifurcation and formation are important and evaluation before operation helps in planning of the anterior surgical approaches of the lumbar vertebrae. Careful dissection of presacral space is essential to minimize complication. Therefore, this study was conducted to aid surgeons who operate for pelvic disorder for careful identification of aorta and sacral promontory before dissection to avoid complication. The specific objectives of this study were to estimate the aorta sacral promontory distance in female patients and to correlate aorta sacral promontory distance with age and BMI.

\section{Methodology}

This was a quantitative, cross-sectional study conducted in a tertiary hospital in Nepal for the period of 4 months. Purposive sampling technique was used in sampling of the subjects. All the subjects who visited for CECT scan of abdomen without pathological findings were included in the study. Measurement was performed on CECT scan of abdomen on sagittal section. Female patients aged 18 and above referred for CECT scan of abdomen without pathological finding were included in the study. Patients with sacral injury, aortic and spinal diseases were excluded in the study. The protocol parameters were as following with triple phase scan delay as $18-20 \mathrm{sec}$ for arterial phase, 45-55 sec for portal phase and 65-70 sec for venous phase (Table 1).

In this study, measurement of aorta sacral promontory was measured by reconstructing arterial phase of the abdomen on sagittal image, point of aortic bifurcation was identified on coronal image and then distance from level of aortic bifurcation to sacral promontory was measured. Vertical distance was represented as aorta sacral promontory distance (Figure 1). Statistical analysis was carried out with the help of SPSS version 25 and Microsoft Excel version 2016. $\mathrm{P}$ value less than 0.01 was considered as level of significance. The mean, SD of APT distance and BMI were expressed in tables and bar diagrams. Data were presented as mean and SD for all variables. 
Patient's age, weight, height was recorded. Data was obtained and analyzed using the descriptive statistics to summarize the information, and inferential statistics (paired sample t-test) to verify if there were significant difference between patient's APT distance with age and BMI.

\section{Table I CECT Protocolin TUTH}

\section{CECT Protocolin TUTH (in Seimens Somatom I 28 slice)}

$.600 \mathrm{ml}$ plain water $30 \mathrm{~min}$ before and then $400 \mathrm{ml}$ just before examination.

-Scanning area from dome of diaphragm to iliaccrest.

-Detect or collimation:0.6x 128

-Pitch: 0.85

$\cdot K v: 120$

•mAs: 200

-Contrast: Intravenous

-Volume: $80-100 \mathrm{ml}$ atrate of $3.5-4 \mathrm{ml} / \mathrm{s}$

-Reconslice and interval:5×5 mm

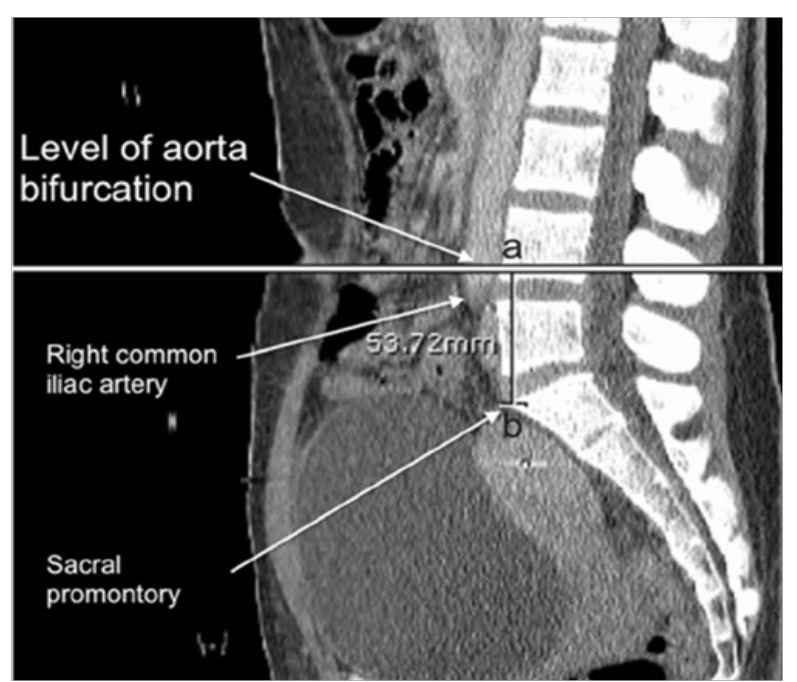

Figure I Measurement of distance from level of aortic bifurcation to sacral promontory. Vertical distance represents the aorta sacral promontory distance.

\section{Results}

Data was collected from 140 subjects who underwent CECT scan for abdomen. Female patients were categorized into four age groups of $18-38,39-59,60-80,80$ and above. The mean age was 48.2 years (range 18-88 years). Total number of patients in age groups of 18-38 years, 39-59 years was 50 each, 60-80 years was 33 and in age group of 80 years and above, number of patients were 7 (Table 2). As the age group increased, there was a decrease in mean APT distance. The mean APT distance was longer among the age group of 18-38 years and obese group. APT distance was short in elderly female patients and underweight patients. Total number of patients in age groups of
18-38 years, $39-59$ years was 50 each, $60-80$ years was 33 and in age group of 80 years and above, number of patients was 7 (Table $3)$. The mean aorta sacral promontory distance of age group 18-38 years was found to be $5.05 \pm 0.56 \mathrm{~cm}, 39-59$ years to be $4.96 \pm 0.57 \mathrm{~cm}$, $60-80$ years to be $3.60 \pm 0.54 \mathrm{~cm}, 80$ years and above to be $2.80 \pm 0.39$ $\mathrm{cm}$. As the age group increased, there was a decrease in mean APT distance. The mean APT distance was longer among the age group of 18-38 years (Table 4). Mean BMI was 23.91 (range 17.11-37.83). The number of patients in underweight group $(\mathrm{BMI}<18.5)$ was 7 and mean APT distance was $17.85 \pm 0.49 \mathrm{~cm}$, normal BMI group (18.5-25) was 88 with mean APT distance $22.4 \pm 1.74 \mathrm{~cm}$, in overweight group (2530 ) was 40 and mean APT was $27.07 \pm 1.48 \mathrm{~cm}$, in the obese group (30 and above) there were 5 patients with mean APT distance $32.97 \pm 3.07$ $\mathrm{cm}$. As BMI increased in patients, there was increase in APT distance (Table 5). There was a strong negative correlation between APT and age. Increased in age was correlated with decrease in APT distance (Pearson's correlation coefficient (r) was found-0.756 between age and APT distance, $\mathrm{p}<0.01)$. There was no significant correlation between BMI and APT distance (Pearson's correlation coefficient (r) was found-0.018 between BMI and APT distance, $p>0.01$ ) (Table 4). Hence, age and BMI were responsible for variation in APT distance. Therefore, there was an overall significant relation between APT distances with age (Figure 2). There was strong negative correlation between APT distance and age (correlation coefficient $\left(r^{2}\right)$ value was found to be 0.572). Increased in age leads to decrease in APT distance (Figure 3). There was no significant correlation between BMI and APT distance (correlation coefficient $\left(\mathrm{r}^{2}\right)$ value was found to be Obetween BMI and APT distance) (Figure 4).

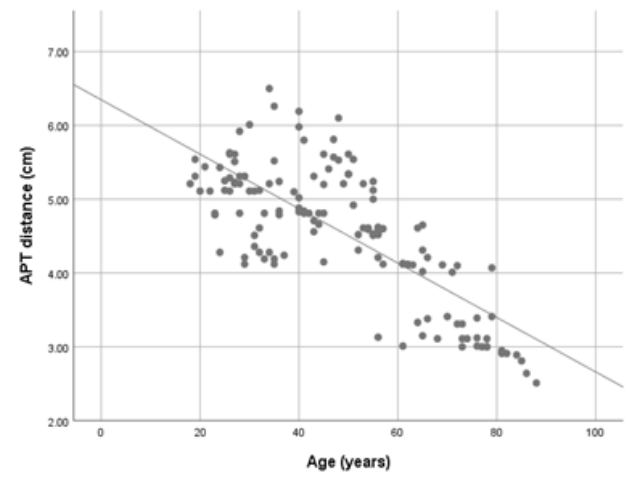

Figure 2 Scattered plot between age in years and APT distance in $\mathrm{cm}$.

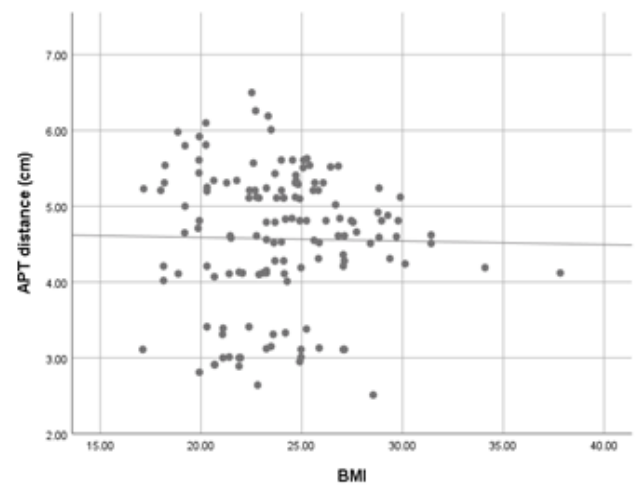

Figure 3 Scattered plot between BMI and APT distance in $\mathrm{cm}$. 


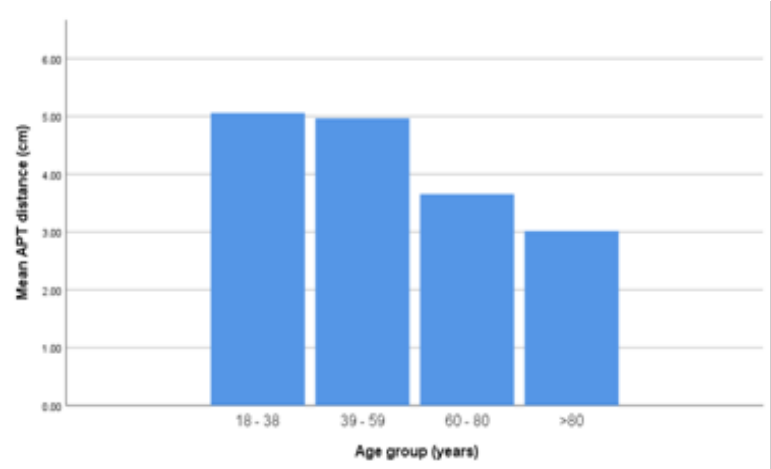

Figure 4 Relationship between age in years and APT distance in $\mathrm{cm}$.

Table 2 Distribution of sample size according to age group

\begin{tabular}{lll}
\hline Age (in years) & Frequency & Percent \\
\hline $18-38$ & 50 & $35.71 \%$ \\
$39-59$ & 50 & $35.71 \%$ \\
$60-80$ & 33 & $23.58 \%$ \\
80 and above & 7 & $5 \%$
\end{tabular}

Table 3 Comparison of mean aorta sacral promontory distance with age

\begin{tabular}{ll}
\hline Age (in years) & $\begin{array}{l}\text { Aorta sacral promontory distance } \\
(\mathbf{c m}) \text { mean } \pm \text { standard deviation }\end{array}$ \\
\hline $18-38$ & $5.05 \pm 0.56$ \\
$39-59$ & $4.96 \pm 0.57$ \\
$60-80$ & $3.60 \pm 0.54$ \\
80 and above & $2.80 \pm 0.39$
\end{tabular}

Table 4 Comparison of mean aorta sacral promontory distance with BMI

\begin{tabular}{lll}
\hline BMI & $\begin{array}{l}\text { Number of } \\
\text { patients }\end{array}$ & $\begin{array}{l}\text { Aorta sacral promontory } \\
\text { distance }(\mathbf{c m}) \text { mean } \pm \\
\text { standard deviation }\end{array}$ \\
\hline Underweight $(<18.5)$ & 7 & $17.85 \pm 0.49$ \\
Normal $(18.5$ to $<25)$ & 88 & $22.4 \pm 1.74$ \\
Overweight (25 to 30) & 40 & $27.07 \pm 1.48$ \\
Obese (30 and above) & 5 & $32.97 \pm 3.07$ \\
\hline
\end{tabular}

Table 5 Linear regression model for predictors of APT distance

\begin{tabular}{lll}
\hline Variable & r-value & p-value \\
\hline Age & -0.756 & $<0.01$ \\
Body mass index & -0.018 & $>0.01$ \\
\hline
\end{tabular}

\section{Discussion}

The number of elderly patients undergoing surgeries for pelvic floor disorders is increasing gradually in recent years. ${ }^{1}$ As more patients are seeking surgical treatment for pelvic prolapse, surgeons should be familiar with proximity of nearby vessels to avoid complications to the iliac artery, iliac veins and aortic bifurcation. Before a surgery, it will be beneficial for surgeons if APT distance is measured using CT scans which can prevent intraoperative complications like injury to vessels, haemorrhage, ureteral injury and enterotomy. This study showed that as the age group increases, there was significant decrease in mean APT distance. However, as BMI increased in patients, there was increase in APT distance but was not significant. APT distance was short in elderly female patients. This result agreed with the result of the study conducted by Sneha Mary Varghese, Suresh Sukumar, Abhimanyu Pradhan. ${ }^{2}$ From 172 subjects the mean age was 56.5 years $(18-95)$ and mean BMI was 23.25 (range 10.8-35.7). The mean APT distance was found to be $2.7 \pm 15 \mathrm{~cm}$. The study also showed that as the age group increased, there was a significant decrease in mean APT distance. However, as the BMI increased in patients, there was no significant change in mean APT distance. The observation in this present study was similar with earlier research conducted by Agrawal A et al. ${ }^{7}$ Mean age was 56.6 years and mean APT distance measured on CT was $6.3 \pm 1.02 \mathrm{~cm}$. This study also supported that within the increasing age group, the mean APT distance significantly decreased. With increase in age there was degeneration of intervertebral discs which shortens the vertebral column and thus a lower level of aortic bifurcation may result. ${ }^{8}$ Major limitations of this study was large number of patients could not be included. We measured patient's parameter manually and the value may not be consistent. We measured the parameters of different patients so the level of the site of measurement might not be the same in all cases. Due to less time period, the number of female patients in BMI group $>30$ and also number of patients in age group $>80$ years was quite less. This type of study should be done in larger number of normal adult population to make the study more reliable.

\section{Conclusion}

The mean age was found to be 48.20 years, mean APT distance $4.57 \pm 0.90 \mathrm{~cm}$ and mean BMI 23.91.This study showed that as the age group increase, there was significant decrease of mean APT. However, with increase in BMI there was no significant change in mean APT.

\section{Acknowledgments}

None.

\section{Funding}

None.

\section{Conflicts of interest}

Author declares that there is no conflict of interest.

\section{References}

1. Nygaard IE, McCreery R, Brubaker L, et al. Abdominal sacrocolpopexy: a comprehensive review. Obstet Gynecol. 2004;104(4):805-823.

2. Varghese S, Sukumar S, Pradhan A. Comparison of Aorta-sacral Promontory Distance with Age and BMI in Female Patients Undergoing CT. Journal of Clinical \& Diagnostic Research. 2017;11(10).

3. Hendrix SL, Clark A, Nygaard I, et al. Pelvic organ prolapse in the Women's Health Initiative: gravity and gravidity. Am J Obstet Gynecol. 2002;186(6):1160-1166.

4. Kornreich L, Hadar H, Sulkes J, et al. Effect of normal ageing on the sites of aortic bifurcation and inferior vena cava confluence: a CT study. Surgical and Radiologic Anatomy. 1998;20(1):63-68.

5. Gurung G, Rana A, Amatya A, et al. Pelvic organ prolapse in rura Nepalese women of reproductive age groups: What makes it so common? Nepal Journal of Obstetrics and Gynaecology. 2007;2(2):35-41. 
6. Good MM, Abele TA, Balgobin S, et al. Vascular and ureteral anatomy relative to the midsacral promontory. Am J Obstet Gynecol. 2013;208(6):486-491.

7. Agrawal A, Abayazeed A, Francis SL, et al. Correlation of patient age with CT-measured aorta-sacral promontory distance. Int Urogynecol J. 2015;26(6):887-891
8. Appaji AC, Kulkarni R, Pai SB. Level of bifurcation of aorta and iliocaval confluence and its clinical relevance. IOSR J Dent Med Sci. 2014;13:5660 . 